\title{
Contribución de colombianos a la generación de conocimiento y desarrollo tecnológico internacional a partir de solicitudes de patentes PCT
}

\section{(Colombians Contribution to the generation of knowledge and international technological development from PCT patent applications)}

\author{
David Romero Betancur ${ }^{1}$, Blanca Janneth Parra Villamil ${ }^{2}$ \\ ${ }^{1}$ Politécnico Internacional, Bogotá, Colombia \\ ${ }^{2}$ Universidad Piloto de Colombia, Bogotá, Colombia \\ jesus.romerob@pi.edu.co,estor-innovaciontec@unipiloto.edu.co
}

\begin{abstract}
Resumen: Uno de los indicadores que permiten medir el desarrollo tecnológico y la innovación de un país es el número de solicitudes de patentes, estas además de brindar protección a los inventores en un territorio específico, potencian el desarrollo económico y fomentan la innovación científica y tecnológica. El estudio que se presenta a continuación está basado en el análisis de los registros de solicitudes de patentes en el marco del Tratado de Cooperación de Patentes (PCT por sus siglas en inglés), en las que han participado como titulares colombianos, y que han sido presentadas durante los últimos 20 años en jurisdicciones diferentes a Colombia. La información fue obtenida de la base de datos de patentes de la Organización Mundial de la Propiedad Intelectual (OMPI). Dentro de los factores analizados se encuentra la dinámica en la que se dio la cooperación entre los titulares y los sectores tecnológicos de mayor tendencia de protección, para esto se empleó un abordaje de tipo cuantitativo con enfoque descriptivo.
\end{abstract}

Los resultados evidencian que los países de mayor interés para tramitar solicitudes de patentes son Europa, Norteamérica y Asia, con una participación mínima en la región centro y suramericana, de igual forma se destaca el trabajo colaborativo que es notorio por el alto número de solicitudes presentadas en co-titularidad, bien sea empresa, universidad, organismo gubernamental o persona natural, tan solo una tercera parte de las solicitudes corresponde a aquellas en cabeza de un solo titular, de las que cerca de la mitad corresponde a personas naturales; este panorama refleja el alto grado de cooperación en torno al cual se generan los desarrollos tecnológicos que relacionan a colombianos.

Palabras clave: Patentes, Desarrollo Tecnológico, Generación de Conocimiento

\begin{abstract}
One of the indicators that make it possible to measure the technological development and innovation of a country is the number of patent applications, these, in addition to providing protection to inventors in a specific territory, enhance economic development and promote scientific and technological innovation. The study presented below is based on the analysis of the records of patent applications within the framework of the Patent Cooperation Treaty (PCT for its acronym in English), in which they have participated as Colombian holders, and that have been presented during the last 20 years in jurisdictions other than Colombia. The information was obtained from the patent database of the World Intellectual Property Organization (WIPO). Among the factors analyzed is the dynamics in which there was cooperation between the holders and the technological sectors with the greatest protection trend, for this a quantitative approach with a descriptive approach was used.
\end{abstract}


The results show that the countries of greatest interest to process patent applications are Europe, North America and Asia, with minimal participation in the Central and South American region, in the same way the collaborative work stands out, which is notorious for the high number of applications submitted. in co-ownership, be it a company, university, government body or natural person, only a third of the applications correspond to those headed by a single owner, of which about half correspond to natural persons; This panorama reflects the high degree of cooperation around which the technological developments that relate to Colombians are generated.

Keywords: Patents, Technological Development, Knowledge Generation

\section{INTRODUCCIÓN}

El sistema de patentes representa una herramienta de alto valor para la identificación de las dinámicas de innovación y brinda un panorama sobre el desarrollo tecnológico de regiones o países específicos, un ejemplo de esto es el del ejercicio llevado a cabo por Kessler y Sperling [1], quienes proponen una visión de la información de las patentes con el fin de establecerla como un framework para el rastreo de procesos de innovación.

Otros autores como Abbas y Khan [2] han adelantado revisiones del estado del arte sobre el análisis de las patentes y el uso de la información contenida en estas para el desarrollo de diferentes estudios, tal es el caso de Ajay y Sangamwar [3] quienes abordaron esta información desde un enfoque del análisis geográfico en países concretos, mientras que con relación a miembros de organismos multilaterales como pueden ser los miembros de la Organización para la Cooperación y el Desarrollo Económicos OCDE se han propuesto ejercicios como los desarrollados por Archontakis y Varsakelis [4], o desde el punto de vista de las empresas el desarrollado por Belderbos [5].

Estos ejercicios demuestran la importancia que representa la información contenida en las patentes para el reconocimiento de las dinámicas propias de innovación y de generación o aprovechamiento de conocimiento en diferentes contextos, por esto en el presente estudio se presentan elementos generales sobre el aporte internacional generado con la participación de colombianos, desde el punto de vista de las patentes solicitadas a nivel mundial, tomando como referente los registros existentes ante la Organización Mundial de la Propiedad Intelectual (OMPI).

\section{METODOLOGÍA}

Con el fin de abordar el alcance que ha tenido el conocimiento aplicado y el desarrollo tecnológico generado por colombianos en el contexto internacional, se toma como referente aquellas solicitudes de patentes, presentadas en el marco de Tratada de Cooperación de Patentes (PCT por sus siglas en inglés) en las que por lo menos uno de los titulares vinculados es colombiano, para esto se utilizó como fuente de información la base de datos de la OMPI, utilizando como estrategia de búsqueda la consulta sobre los registros que asocian la nacionalidad del titular con Colombia, para el periodo comprendido entre los años 2000 y 2019.

El análisis de esta información se centró en la identificación de los principales titulares, la dinámica en la que se dio la cooperación entre ellos y los sectores tecnológicos relacionados según los registros, para lo cual se empleó un abordaje cuantitativo con enfoque descriptivo [6], centrado en el comportamiento general de las solicitudes identificadas.

Previo al análisis de la información se realizó la normalización de los datos, especialmente en cuanto a los nombres de titulares asociados, a fin de facilitar la identificación del tipo al que corresponden según sean empresa, universidad, persona natural o ente gubernamental, y evitar registros diferentes de un mismo titular. Para las solicitudes identificadas se tomó como registro 
independiente cada clase correspondiente a la Clasificación Internacional de Patentes (IPC por su sigla en inglés), que tiene asociada.

De esta forma se identificaron en cada solicitud los titulares y las clases que relaciona, permitiendo así obtener dos registros independientes: inicialmente los solicitantes y los registros asociados a cada uno [7], facilitando el reconocimiento del tipo de persona; y por otro lado la relación de las clase de patentes vinculadas a cada solicitud de forma independiente y relacionada con cada registro [8], para así tener claridad de su frecuencia de aparición.

De conformidad con esta metodología a continuación se presentan los aspectos generales de los resultados obtenidos.

\section{RESULTADOS}

Para la ventana de análisis de solicitudes de patentes de los últimos 20 años (2000 a 2019), se obtuvo un total de 2.267 registros que vinculan por lo menos a un colombiano entre sus titulares, igualmente se identificó que estos asocian en total a 5.317 titulares, de los cuales el $84 \%$ corresponde a personas naturales, el $12,7 \%$ a empresas, el $3,1 \%$ a universidades y el $0,2 \%$ a entidades gubernamentales.

En relación con la dinámica de cooperación se evidenció que el 31\% de las solicitudes son presentadas en cabeza de un solo titular, mientras que el 69\% son presentadas de forma conjunta por titulares de diferente tipo según los grupos de interés, como se presenta en la Figura 1, lo que evidencia el fuerte comportamiento del trabajo colaborativo para el desarrollo de invenciones.

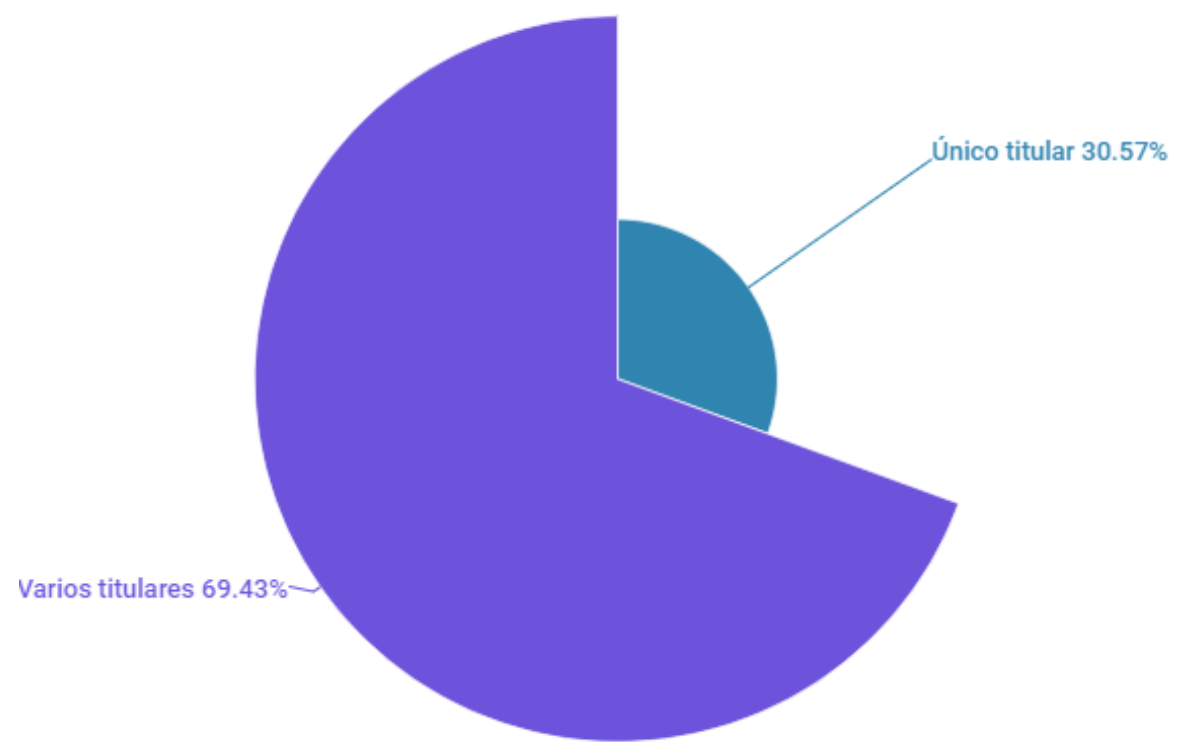

Figura 1. Distribución de solicitudes que asocian uno o varios tipos de titulares

Elaboración propia, fuente de información base de datos de patentes de la OMPI (patentscope.wipo.int) abril 1 de 2020

Este comportamiento identificado evidencia que la gran mayoría de colombianos relacionados con solicitudes de patente presentadas en países diferentes al suyo de origen, lo hacen en asocio con por lo menos otra persona, bien sea natural o jurídica. 
En relación con el sector tecnológico, analizado a partir de la clasificación internacional de patentes, se identificó que 1.319 registros, correspondiente al 58\% del total de solicitudes de la ventana de observación, están asociados a la clase A61P 35/00 "Agentes antineoplásicos", en la Tabla 1 se relacionan las 10 clases con mayor cantidad de solicitudes relacionadas y el porcentaje que estas representan del total identificadas.

Tabla 1. Principales IPC asociadas a las patentes que vinculan a colombianos

\begin{tabular}{|c|c|c|c|}
\hline Clase & Nombre & $\begin{array}{l}\text { Total } \\
\text { Solicitudes }\end{array}$ & $\%$ \\
\hline A61P 35/00 & Agentes antineoplásicos & 1319 & $58 \%$ \\
\hline A61K 39/395 & $\begin{array}{l}\text { Preparaciones medicinales que contienen antígenos } \\
\mathrm{o} \text { anticuerpos } \bullet \text { Anticuerpos }\end{array}$ & 642 & $28 \%$ \\
\hline C07D 471/04 & $\begin{array}{l}\text { Compuestos heterocíclicos que contienen átomos de } \\
\text { nitrógeno como únicos heteroátomos del sistema } \\
\text { condensado, teniendo al menos un ciclo de seis } \\
\text { miembros con un átomo de nitrógeno, no previstos } \\
\text { en los grupos } \bullet \text { Sistemas condensados en orto }\end{array}$ & 573 & $25 \%$ \\
\hline C07D 487/04 & $\begin{array}{l}\text { Compuestos heterocíclicos que contienen átomos de } \\
\text { nitrógeno como únicos heteroátomos del ciclo en el } \\
\text { sistema condensado } \cdots \text { Sistemas condensados en } \\
\text { orto }\end{array}$ & 562 & $25 \%$ \\
\hline A61P 25/00 & $\begin{array}{l}\text { Medicamentos para el tratamiento de trastornos del } \\
\text { sistema nervioso }\end{array}$ & 496 & $22 \%$ \\
\hline A61P 29/00 & $\begin{array}{l}\text { Agentes analgésicos, antipiréticos o } \\
\text { antiinflamatorios que no actúan sobre el sistema } \\
\text { nervioso central, p. ej. agentes antirreumáticos; } \\
\text { Antiinflamatorios no esteroideos (AINEs) }\end{array}$ & 465 & $21 \%$ \\
\hline C07K 16/28 & $\begin{array}{l}\text { Inmunoglobulinas, p. ej. Anticuerpos mono o } \\
\text { policlonales } \bullet \cdot \text { contra receptores, antígenos } \\
\text { celulares de superficie o determinantes celulares de } \\
\text { superficie }\end{array}$ & 456 & $20 \%$ \\
\hline C07D 401/14 & $\begin{array}{l}\text { Compuestos heterocíclicos que contienen dos o más } \\
\text { heterociclos, que tienen átomos de nitrógeno como } \\
\text { únicos heteroátomos del ciclo, siendo al menos un } \\
\text { ciclo de seis miembros con solamente un átomo de } \\
\text { nitrógeno } \bullet \text { que contienen tres o más heterociclos }\end{array}$ & 425 & $19 \%$ \\
\hline A61F 13/15 & $\begin{array}{l}\text { Vendas o apósitos - Compresas absorbentes, p. ej. } \\
\text { paños higiénicos, torundas o tampones para } \\
\text { aplicación externa o interna al cuerpo. Medios para } \\
\text { mantenerlos en su sitio o para fijarlos; Aplicadores } \\
\text { de tampone }\end{array}$ & 422 & $19 \%$ \\
\hline
\end{tabular}

Según los datos analizados se encuentra que el 28,2\% de las solicitudes identificadas se encuentran relacionadas con el sector de productos farmacéuticos, específicamente en medicamentos relacionados con sustancias que impiden el desarrollo, crecimiento, o proliferación de células tumorales malignas, así como anticuerpos propiamente dichos, antiinflamatorios y medicamentos para trastornos nerviosos.

La única clase identificada que no tiene relación directa con productos farmacéuticos corresponde a A61F 13/15, relacionada con vendas o apósitos, relacionados principalmente con 
elementos de aseo femenino. Si bien estos últimos no tienen relación directa con productos farmacéuticos, su relación con los productos médicos o de salud si es directa.

De acuerdo con esta clasificación y tomando como referencia la Tabla 2 donde se presentan las concordancias con la clasificación internacional de patentes de la Oficina Española de Patente y [9], se identifican los sectores más relevantes según los registros analizados.

Tabla 2. Concordancia de IPC

\begin{tabular}{l|l} 
Sector tecnológico & Subsectores \\
\hline Electricidad, & Aparatos electrónicos, Ingeniería Electrónica, Ingeniería Eléctrica \\
electica & Comunicación Digital \\
& Métodos de gestión mediante tecnologías de la información \\
& Procesos básicos de comunicación \\
Semiconductores \\
Tecnología Audiovisual \\
& Tecnología Informática \\
& Telecomunicaciones \\
Química & Análisis de materiales biológicos \\
& Control \\
& Medida \\
& Optica \\
& Tecnología médica \\
& Biotecnología \\
& Ingeniería química \\
& Materiales, metalurgia \\
& Productos farmacéuticos \\
& Productos orgánicos elaborados \\
& Química de alimentos \\
& Química de materiales \\
& Química macromolecular, polímeros \\
& Tecnología de las microestructuras nanotecnología \\
& Tecnología de superficie, revestimiento \\
& Tecnología medioambiental \\
Ingeniería Mecánica & Componentes mecánicos \\
& Manipulación \\
& Maquinaria textil y de papel \\
& Máquinas herramienta \\
& Motores, bombas, turbinas \\
& Otra maquinaria especial \\
& Procesos térmicos y aparatos \\
& Transporte \\
& Ingeniería civil \\
& Mobiliario y juegos \\
& Otros productos de consumo \\
&
\end{tabular}

Se observa que el sector en el que se encuentra mayor participación corresponde al de productos farmacéuticos (Química), en el que además el mayor número de solicitudes se presentó en los años 2009 y 2011, seguido por el de tecnología médica (Instrumentos); cada uno de estos dos sectores están relacionados en el $28 \%$ de las solicitudes presentadas en el periodo de interés, otro sector de alto nivel de protección es el de química de materiales. En la Figura 2 
se presenta la distribución de solicitudes según el sector tecnológico para este grupo de patentes entre los años 2000 y 2019.

Desde este punto de vista se refuerza el hallazgo presentado al observar de forma independiente las IPC relacionadas a cada solicitud, encontrando además que en términos del comportamiento año a año, los productos farmacéuticos han representado un interés permanente entre los años 2000 y 2019, mientras que sectores como el de ingeniería civil que en cifras globales representa un escaso interés, ha presentado un incremento sostenido en el total de solicitudes desde los años 2012 y 2013.

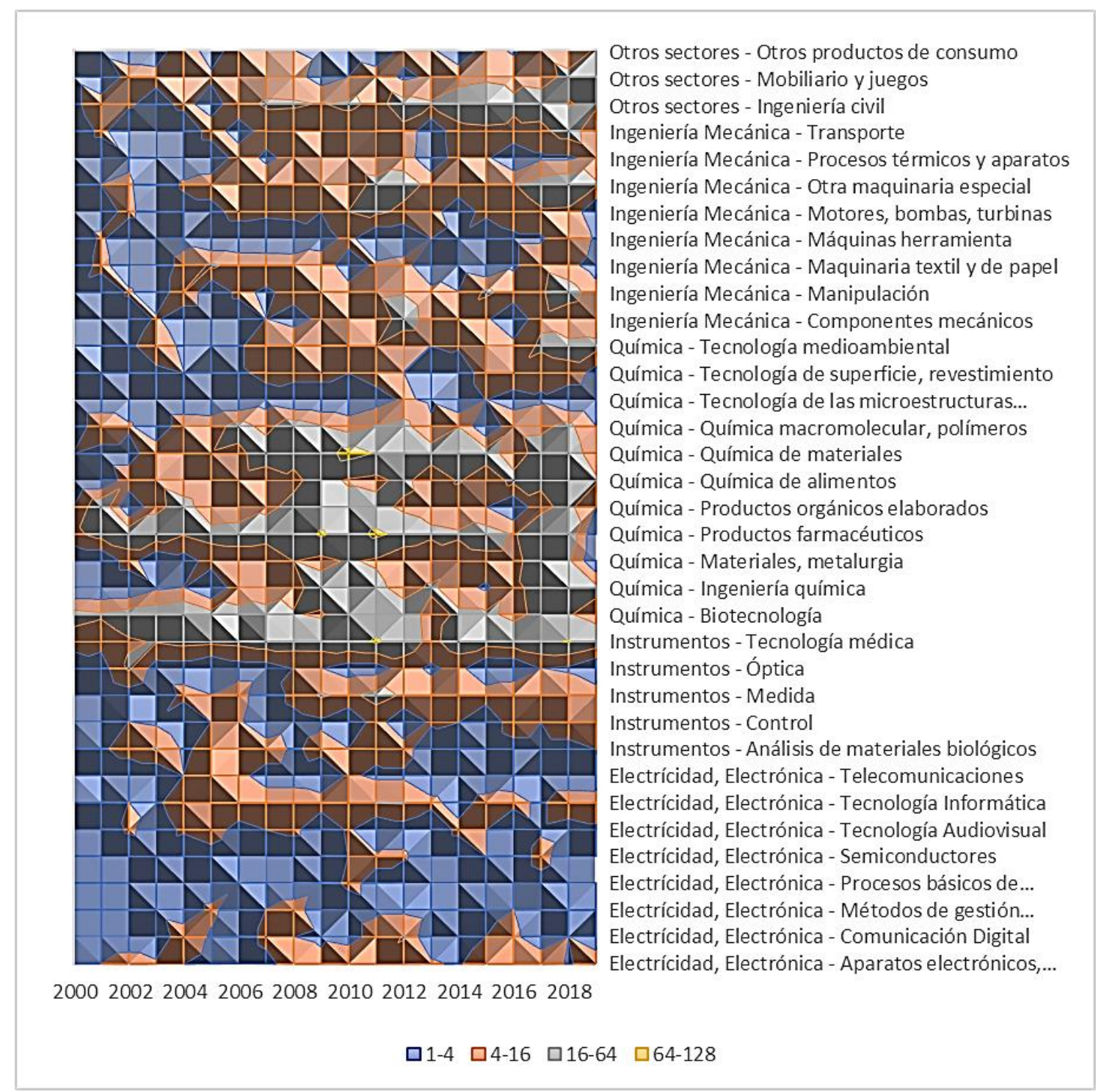

Figura 2. Solicitudes de patente presentadas en el exterior que asocian colombianos, según sector tecnológico asociado por año de solicitud

Elaboración propia, fuente de información base de datos de patentes de la OMPI (patentscope.wipo.int) abril 1 de 2020

Con esta perspectiva también resulta interesante el hecho de que los sectores relacionados con electricidad y electrónica corresponden a aquellos en los que se presenta una menor 
vinculación de titulares colombianos, hecho que contrasta con el comportamiento de las solicitudes de patentes a nivel mundial donde se encuentra que el $24,5 \%$ del total de registros se presentan en estos sectores, liderados por empresas como Canon y Samsung [10], en la Figura 3 se presenta el comportamiento internacional del total de solicitudes presentadas para los años 2007,2012 y 2017.

\begin{tabular}{|c|c|c|c|c|c|}
\hline & & \multicolumn{3}{|c|}{ Number of published applications } & \multirow{2}{*}{$\begin{array}{r}\text { Share of } \\
\text { total }(\%) \\
2017\end{array}$} \\
\hline \multicolumn{2}{|c|}{ Field of technology } & 2007 & 2012 & 2017 & \\
\hline \multirow{8}{*}{$\begin{array}{l}\text { Electrical } \\
\text { engineering }\end{array}$} & Electrical machinery, apparatus, energy & 102,410 & 146,626 & 197,645 & 6.7 \\
\hline & Audio-visual technology & 93,742 & 77,319 & 82,888 & 2.8 \\
\hline & Telecommunications & 66,954 & 51,694 & 58,467 & 2.0 \\
\hline & Digital communication & 64,059 & 91,738 & 144,669 & 4.9 \\
\hline & Basic communication processes & 17,794 & 16,345 & 16,685 & 0.6 \\
\hline & Computer technology & 125,073 & 150,721 & 229,269 & 7.8 \\
\hline & IT methods for management & 20,414 & 28,743 & 53,326 & 1.8 \\
\hline & Semiconductors & 80,228 & 85,794 & 83,954 & 2.8 \\
\hline \multirow[t]{5}{*}{ Instruments } & Optics & 78,025 & 65,003 & 73,134 & 2.5 \\
\hline & Measurement & 66,697 & 94,890 & 148,809 & 5.0 \\
\hline & Analysis of biological materials & 11,354 & 12,440 & 17,869 & 0.6 \\
\hline & Control & 27,776 & 32,997 & 67,309 & 2.3 \\
\hline & Medical technology & 75,479 & 89,164 & 132,863 & 4.5 \\
\hline \multirow[t]{11}{*}{ Chemistry } & Organic fine chemistry & 54,696 & 55,306 & 68,901 & 2.3 \\
\hline & Biotechnology & 34,623 & 43,222 & 64,012 & 2.2 \\
\hline & Pharmaceuticals & 75,046 & 75,788 & 106,312 & 3.6 \\
\hline & Macromolecular chemistry, polymers & 28,444 & 33,631 & 54,504 & 1.8 \\
\hline & Food chemistry & 21,262 & 34,580 & 74,470 & 2.5 \\
\hline & Basic materials chemistry & 39,717 & 54,239 & 95,776 & 3.2 \\
\hline & Materials, metallurgy & 30,734 & 48,464 & 71,684 & 2.4 \\
\hline & Surface technology, coating & 30,091 & 38,879 & 46,696 & 1.6 \\
\hline & Micro-structural and nano-technology & 2,594 & 4,295 & 5,294 & 0.2 \\
\hline & Chemical engineering & 33,888 & 44,848 & 80,378 & 2.7 \\
\hline & Environmental technology & 21,900 & 32,006 & 55,918 & 1.9 \\
\hline \multirow{8}{*}{$\begin{array}{l}\text { Mechanical } \\
\text { engineering }\end{array}$} & Handling & 43,261 & 51,316 & 85,296 & 2.9 \\
\hline & Machine tools & 37,130 & 56,168 & 89,742 & 3.0 \\
\hline & Engines, pumps, turbines & 42,149 & 56,113 & 65,948 & 2.2 \\
\hline & Textile and paper machines & 36,316 & 34,849 & 44,541 & 1.5 \\
\hline & Other special machines & 44,917 & 61,862 & 117,901 & 4.0 \\
\hline & Thermal processes and apparatus & 25,598 & 34,503 & 50,357 & 1.7 \\
\hline & Mechanical elements & 44,321 & 54,196 & 77,156 & 2.6 \\
\hline & Transport & 65,707 & 79,069 & 124,203 & 4.2 \\
\hline \multirow[t]{4}{*}{ Other fields } & Furniture, games & 44,773 & 48,281 & 77,522 & 2.6 \\
\hline & Other consumer goods & 32,227 & 38,997 & 58,809 & 2.0 \\
\hline & Civil engineering & 53,279 & 67,884 & 105,322 & 3.6 \\
\hline & Unknown & 40,822 & 30,963 & 22,976 & 0.8 \\
\hline \multicolumn{2}{|l|}{ Total } & $1,713,500$ & $2,022,933$ & $2,950,605$ & 100.0 \\
\hline
\end{tabular}

Figura 3. Solicitudes de patente publicadas en todo el mundo por sector tecnológico, 2007, 2012 y 2017

Tomado de [10, p. 40]

Con base en esta información también se encuentra que para este grupo los sectores que asocian la menor cantidad de solicitudes son los relacionados con electricidad y electrónica, especialmente la de procesos básicos de comunicación, así como el de máquinas herramienta, en los cuales además ha habido años en los que no se han presentado solicitudes. 
Con el fin de ampliar el análisis de los datos obtenidos y procesados en el marco de la investigación, la información relacionada con el detalle de las solicitudes por año, así como las jurisdicciones asociadas con cada uno de los registros, serán abordados por los autores en posteriores artículos.

\section{CONCLUSIONES}

Conforme a los resultados obtenidos, se encuentra que el conocimiento generado a nivel mundial que vincula a colombianos se centra especialmente en el sector farmacéutico, caracterizándose además porque no es desarrollado de forma independiente sino en alianza con otros inventores.

Otro aspecto para destacar es el alto número de solicitudes de patentes provenientes de personas naturales en contraste con la escasa representación de las universidades quienes son las llamadas a generar conocimiento científico.

En comparación con el contexto internacional, se encuentra que el conocimiento generado desde un el punto de vista científico-tecnológico ofrecido por las patentes, se centra especialmente en el sector tecnológico de productos y procesos eléctricos y electrónicos, mientras que para el caso particular de aquel en el que se vincula a colombianos, sus aportes se centran en el sector químico, con especial interés en el farmacéutico.

\section{REFERENCIAS}

[1] J. Kessler and D. Sperling, "Tracking U.S. biofuel innovation through patents," Energy Policy, vol. 98, pp. 97-107, 2016.

[2] A. Abbas, L. Zhang, and S. U. Khan, "A literature review on the state-of-the-art in patent analysis," World Pat. Inf., vol. 37, pp. 3-13, 2014.

[3] D. Ajay and A. T. Sangamwar, "Identifying the patent trend, licensing pattern and geographical landscape analysis of the council for scientific \& industrial research (CSIR) of India between 2000 and 2011," World Pat. Inf., vol. 38, pp. 42-49, 2014.

[4] F. Archontakis and N. C. Varsakelis, "Patenting abroad: Evidence from OECD countries," Technol. Forecast. Soc. Change, vol. 116, pp. 62-69, 2017.

[5] R. Belderbos, "Overseas innovations by Japanese firms: An analysis of patent and subsidiary data," Res. Policy, vol. 30, no. 2, pp. 313-332, 2001.

[6] R. Hernandez Sampieri, C. Fernández Collado, and M. del P. Baptista Lucio, Metodología de la Investigación, sexta edición., 6th ed. 2014.

[7] J. D. Romero-Betancur and B. J. Parra Villamil, "Solicitantes de patente ante la WIPO (relacionados con solicitudes que vinculen por lo menos a un colombiano)." Zenodo, 2020.

[8] J. D. Romero-Betancur and B. J. Parra Villamil, "Sectores Tecnológicos asociados a las solicitudes de patente ante la WIPO que vinculen por lo menos a un colombiano." Zenodo, 2020.

[9] Oficina Española de Patente y Marcas, "Tabla de concordancia con la Clasificación Internacional de Patentes (CIP)." Oficina Española de Patentes y Marcas, Madrid, pp. 1-8, 2014.

[10] World Intellectual Property Organization, World Intellectual Property Indicators 2019. 2019. 